UDC: $811.134 .2 ' 342.2$

DOI: https://doi.org/10.18485/beoiber.2019.3.1.1

\author{
César Luis Díez Plaza' \\ Instituto Cervantes, Belgrado \\ Serbia
}

\title{
LA NOTACIÓN CIENTÍFICA COMO OBJETO DE ESTUDIO: EL CASO DE <AE> EN LA FONOLOGÍA DE ALARCOS (1950-1965)
}

\begin{abstract}
Resumen
El presente trabajo estudia un caso concreto de notación en la Fonología de Alarcos (1950-1965), el ejemplo de <ae>. El estudio de este glifo permite presentar un modelo de análisis de la notación empleada en los trabajos fonológicos.

Dicho modelo se basa en la relación entre dos dimensiones - la dimensión de los textos y la de la notación científica - que se establece gracias al empleo de dos operaciones: la operación de transcripción y la de transliteración. Además de reflexionar sobre la importancia del estudio de la notación, el objetivo final de este trabajo es proponer que los propios textos científicos (sobre fonología en este caso) se conviertan en un objeto de estudio propio.
\end{abstract}

Palabras clave: teoría de la representación, notación, transliteración, transcripción, glifos, caracteres, símbolos.

\section{SCIENTIFIC NOTATION AS A STUDY OBJECT - THE CASE OF <AE > IN THE PHONOLOGYOF ALARCOS (1950-1965)}

\section{Summary}

The present work studies a concrete case of notation in Alarcos's book Fonología Española (19501965) - the example of <ae>. The study of this glyph allows us to present a model of analysis of systems of notation used in phonological works.

This model is based on the relationship between two dimensions - the dimension of texts and the dimension of scientific notation - which is established through the use of two operations: transcription and transliteration. In addition to reflecting on the importance of the study of notation, the final objective of this work is to propose that the scientific texts themselves (on phonology in this case) become an object of study by themselves.

Key words: representation theory, notation, transliteration, transcription, glyphs, characters, symbols.

\footnotetext{
1 cdplaza@cervantes.es
} 
El objetivo de este trabajo es demostrar la importancia del estudio de la notación para la comprensión e interpretación de las teorías fonológicas propuestas en un momento histórico determinado. Dichas teorías, normalmente, se transmiten en formato escrito, por lo que los textos que componen este corpus teórico son susceptibles de convertirse, a su vez, en un objeto de estudio. Un objeto de estudio que, en esta contribución, se denominará, de manera general, como la dimensión gráfica de la notación científica y se abreviará por medio de las siglas NC colocadas entre corchetes angulares: $<\mathrm{NC}>$.

El ejemplo de $<\mathrm{NC}>$ que va a ser analizado aquí es un párrafo de la obra Fonología española de Emilio Alarcos (1922-1988); una obra que según nos dice Francisco J. Perea (2014: 562):

[...] tuvo cuatro ediciones con notables variaciones entre unas y otras. Sin embargo, en la bibliografía sobre materia fonológica se suele tomar este libro como una obra en cierto modo monolítica, citándose bien por la postrera edición, de 1965 o cualquiera de sus reimpresiones posteriores.

La importancia del texto en cuestión para la historiografía lingüística española (con él se ha formado un gran número de estudiantes en sus 68 años de existencia) y el hecho de que haya tenido una dilatada vida editorial, como demuestra la circunstancia de que su cuarta y última edición se haya reimpreso muchas veces (la manejada para escribir este trabajo es la octava de 1991 y la última localizada es de 2015), son factores que justifican la elección de este texto como caso de análisis. La importancia del propio texto en momento muy cercano al de su concepción y primera redacción la ponen de relevancia las siguientes afirmaciones de J. H. Matluck (1995: 277) con las que abría la reseña que hacía a la segunda edición: "Redactado entre 1948 y 1949, se publicó en 1950 este manual de fonología española, el primero que se ha escrito".

\section{El párrafo elegido y la presentación del texto}

A continuación se citará el párrafo que se va a comentar, tal y como aparece en la primera y en la cuarta edición (1950 y 1965). Dicho párrafo es nuestra dimensión gráfica, la primera con la que se encuentra un lector al entrar en el mundo teórico de la lingüistica; es el "soporte físico" que supone cualquier página de un texto científico (diccionario, gramática, edición crítica, artículo, etc.). 


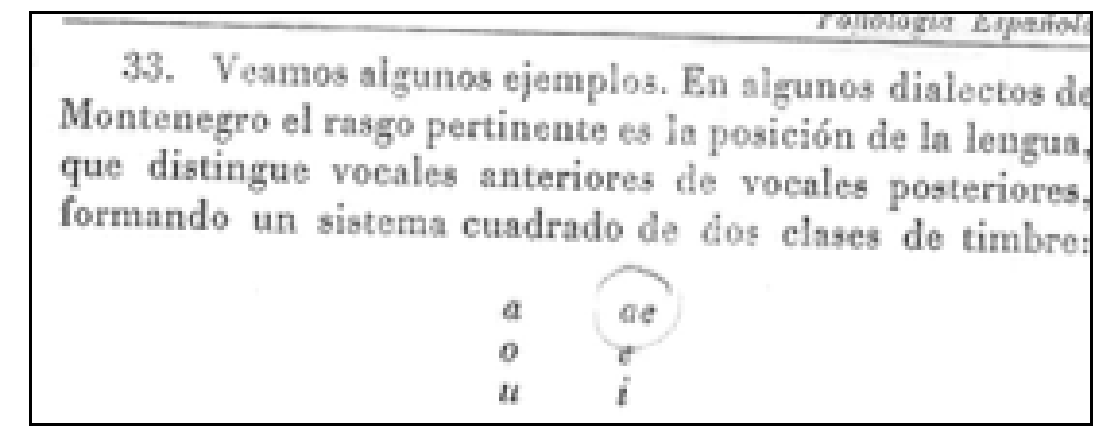

Texto de Alarcos 1: 1ª edición (1950: 40)

33. Veamos algunos ejemplos. En algunos dialectos de Montenegro, el rasgo pertinente es la posición de la lengua, que distingue vocales anteriores (agudas) de vocales posteriores (graves), formando un sistema cuadrado de dos cla. ses de color:

$\begin{array}{cc}u & i \\ \circ & e \\ a & a e\end{array}$

Texto de Alarcos 2: 4⿳亠丷a edición Alarcos (1965: 61)

Como una decisión metodológica, en este trabajo se incluirán imágenes escaneadas del corpus de textos de la $\langle\mathrm{NC}\rangle$ utilizados en vez de recurrir a copiar el contenido de dichos textos. Esta decisión permite confrontarse con los textos originales, con las tipografías y distribuciones gráficas que eligieron los autores o editores, en vez de leerlos transcritos a la fuente y distribución gráfica específica de este trabajo. De esta manera, el presente trabajo se puede concebir también como un elemento de la $\langle\mathrm{NC}\rangle$, en el que se intenta distinguir claramente entre la parte expositiva del trabajo y la referencia a otras $<\mathrm{NC}>$ utilizadas como ejemplos o argumentos.

La redacción del texto de ambas ediciones (la de 1950 y la de 1965) presenta una evolución en el contenido. Se completa la descripción de términos como "vocales anteriores" y "vocales posteriores" con otros usos teóricos de términos como "agudas" y "graves", además de sustituir el término "timbre" por el de "color" (aunque este último siga apareciendo entre paréntesis en otros párrafos del texto de la cuarta edición). Un análisis de este tipo de diferencias sería muy interesante para ver la evolución del planteamiento teórico del autor que se upreocupa por perfeccionar la obra que comenzó en 1950" (Perea 2014: 570). Un punto de vista que pertenecería al campo de la historia (o la historiografía) de la lingüística, enfoque en el que se incluye el volumen colectivo en el que aparece la obra de Perea (Calero et al. 2014). Sin embargo, desde la óptica de este trabajo, que se incluiría en una teoría general de la representación o la notación, lo 
interesante reside precisamente en lo que no cambia: la notación de ese sistema cuadrado de seis vocales que se identifica en algunos dialectos de Montenegro.

Una enigmática referencia geodialectal, que habla de dialectos sin citar específicamente la lengua a la que pertenecen; pero que, sin embargo, permite localizar la fuente que ha servido de inspiración al autor: el libro de Trubetzkoy Grundzüge der Phonolologie (1938). Aunque, más concretamente, es posible que Alarcos también usase una traducción al francés del mismo, Principes de phonologie, realizada por J. Cantineau en 1947 (aunque para este trabajo se haya manejado una edición posterior de dicha traducción, fechada en 1967) que cuenta con un prólogo de André Martinet y que aparece incluida en la bibliografía que el autor incluyó en la cuarta edición de su Fonología española (1965). A continuación, se reproducirán ambos párrafos - original en alemán y traducción al francés - junto con un tercer párrafo que pertenece a una traducción mucho más reciente (2016) al serbio² del texto alemán de Trubetzkoy; aunque en este caso, tal y como se indica en el libro, la traducción no se hace desde la edición de 1939 (edición póstuma, ya que el autor fallece en 1938) sino desde una edición posterior de 1958.

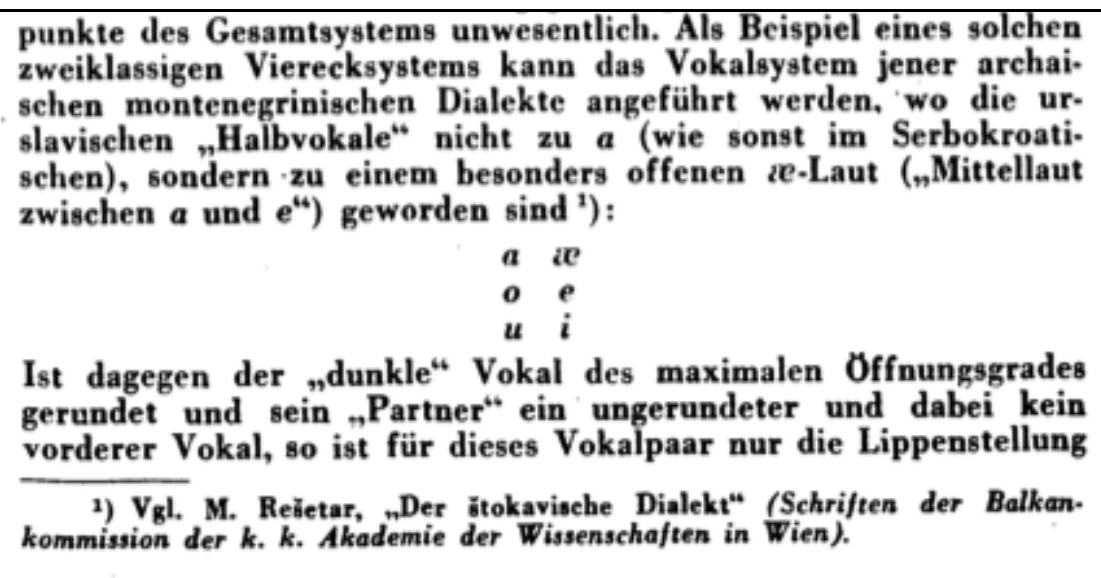

Texto de Trubetzkoy (1939: 89)

2 Existe también una traducción al español de esta obra de Trubetzkoy realizada por García Gordiano (1973) que, desgraciadamente, no se ha podido localizar para incluirla en el corpus de textos analizados en este trabajo. 
bilatérale proportionnelle. Par contre le fait que les voyelles postérieures d'aperture non maxima sont arrondies n'est pas essentiel au point de vue du système. Comme exemple d'un tel système quadrangulaire on peut alléguer le système vocalique de ces dialectes monténégrins où les « semi-voyelles du vieux-slave sont devenues non pas $a$ (comme d'habitude en serbo-croate), mais un $x$ particulièrement ouvert (intermédiaire entre $a$ et $e)^{1}$ :

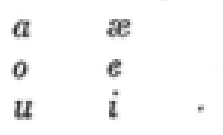

(1) M. Resetar, = Der Stokavisehe Dialekt = (Sehriften der Balkankommisaion der $k, k$. Akademie der Wissenschaflen in Wien).

Texto de Cantineau (Trubetzkoy 1967: 104)

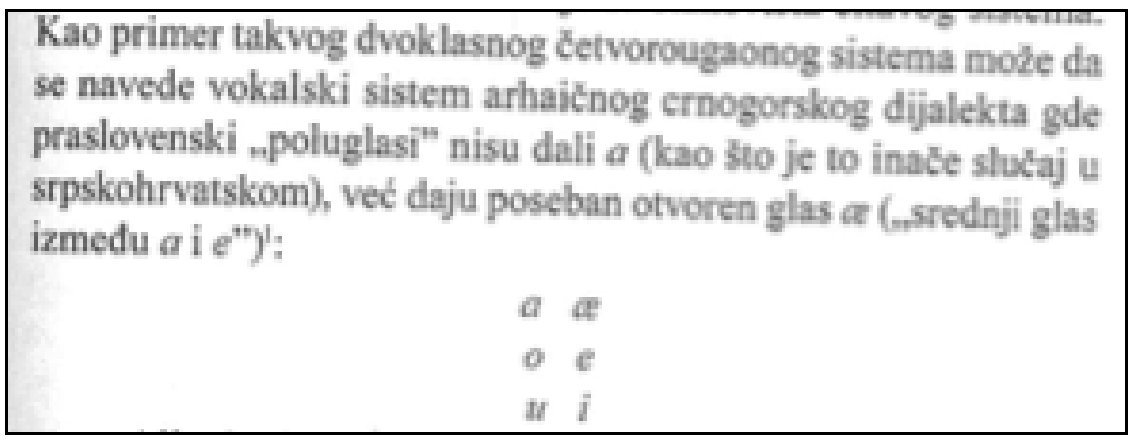

Texto de Berić y Berić (Trubeckoj 2016: 107)

Una diferencia interesante entre la traducción francesa y la serbia es que la segunda mantiene el adjetivo "arcaico" que en el original se ha aplicado a "algunos dialectos montenegrinos", "[sistem] arhaičnog crnogorskog dialekta" por "jener archaisen montenegrinischen Dialekte"; mientras que en la traducción francesa no aparece, "ces dialectes monténégrins". Esa omisión, junto con el uso del plural (frente al singular del texto serbio), hacen pensar que el texto principalmente manejado por Alarcos fuera el de la traducción francesa ya que él dice "[e]n algunos dialectos de Montenegro", plural y sin denominación de "arcaicos".

Por lo que se refiere a la notación usada para las "vocales" coinciden el texto alemán (Trubetzkoy 1939) y sus traducciones al francés (Trubeckoy 1967) y serbio (Trubeckoj 2016); pero difiere de la del texto en español (Alarcos 1950; Alarcos 1965) y no sólo por la distribución gráfica empleada. Por alguna razón, en ese texto español, el orden de la tres filas aparece cambiado a lo largo de los años que distan entre la primera y la última edición: en la edición de 1950, las tres filas están ordenadas, al igual que en la fuente alemana, siguiendo el grado de abertura en sentido de mayor abertura a menor ( $a$ 
- o - u / ae - e - i); mientras que en la última edición (1965) el orden se invierte, apareciendo de menor a mayor abertura $(u-0-a / i-e-a e)$. Es muy posible que esta diferencia sea únicamente de disposición gráfica en la composición del texto y no comporte ningún tipo de variación en la interpretación teórica. Algo que, precisamente, no ocurre con otra diferencia gráfica de mucho mayor calado teórico: el uso de la ligadura $<æ>$ en el caso del original alemán y de su traducción al francés, frente a las dos "letras" separadas que aparecen en el texto español, <ae>.

Pero antes de profundizar en esta polémica, que es el objeto principal de este trabajo, es necesario añadir algo más a la presentación que se ha hecho de los párrafos pertenecientes a los textos en alemán, francés y serbio. Las citas de los párrafos alemán y francés terminan con un número "1" que remite a una nota a pie de página en la que se cita la referencia bibliográfica que Trubetzkoy usa para el caso de la dialectología del serbo-croata: la obra de Milan Rešetar Der stokavische Dialekt (1907)³. Curiosamente, en dicha obra el autor usa una notación distinta para los reflejos en serbo-croata de las "semiconsonantes" del antiguo eslavo, la notación $<\mathrm{a}^{\mathrm{e}}>$, aunque usando la cursiva $\left(a^{e}\right)$, como es uso general en todas las obras citadas. La referencia a Rešetar y el contraste en la notación suscitan el interés por esos dialectos montenegrinos que tan enigmáticamente se citaban en la Fonología Española y una buena fuente para continuar la investigación es una obra coetánea del proceso de redacción de la Fonología, el libro de Pavle lvić Die serbokroatichen Dialekte (1958); aunque, para este trabajo, se haya usado una versión de 1994 que está escrita en serbio usando el alfabeto cirílico ${ }^{4}$.

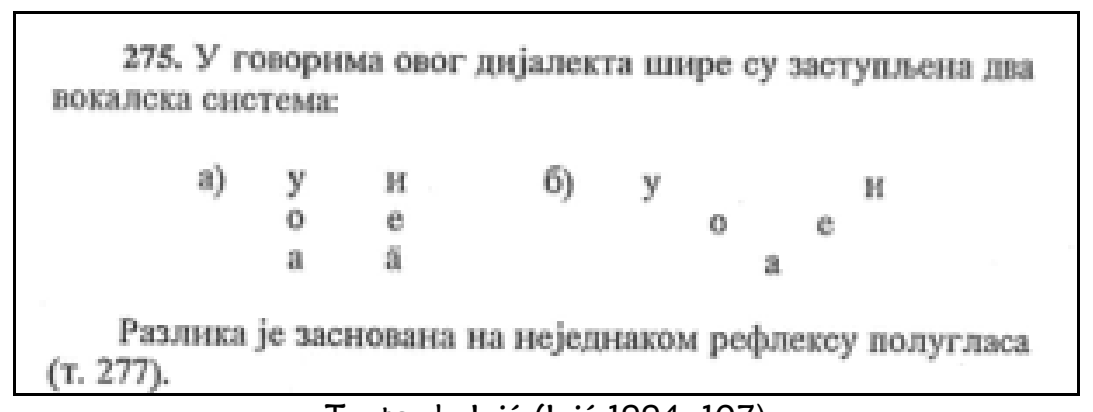

Texto de Ivić (Ivić 1994: 107)

Para el desarrollo de este trabajo, de los dos sistemas recogidos en la obra de lvić, el relevante es el etiquetado con la letra a) que, además, coincide en su presentación gráfica (abertura de menor a mayor) con el que aparece en la cuarta edición de la obra de Alarcos (1965) y que modifica el orden que aparecía en la primera edición de 1950.

${ }^{3}$ En el caso del texto en serbio también aparece la misma cita bibliográfica (p. 107) aunque por razones de espacio (disposición gráfica del texto de este trabajo) no ha aparecido en la imagen del texto presentada.

${ }^{4}$ La traducción del texto serbio es la siguiente: “275. En hablas de este dialecto se encuentran representados ampliamente dos sistemas: [dos esquemas de vocales señalados por a) y b)]. La diferencia entre ellos se fundamenta en un desigual reflejo de la semivocal". 
Sin embargo, y sin entrar por el momento en el tema que supone la operación de transliteración ya que, como se ha dicho, el libro está escrito en alfabeto cirílico, en la obra de lvić se usa una notación diferente para el elemento estudiado: <ä>. Una notación que, paradójicamente, también aparece en la obra de Trubetzkoy (diez páginas después del párrafo que ha dado origen a esta reflexión) para referirse al mismo fenómeno como se muestra a continuación en su original alemán de $1939^{5}$.

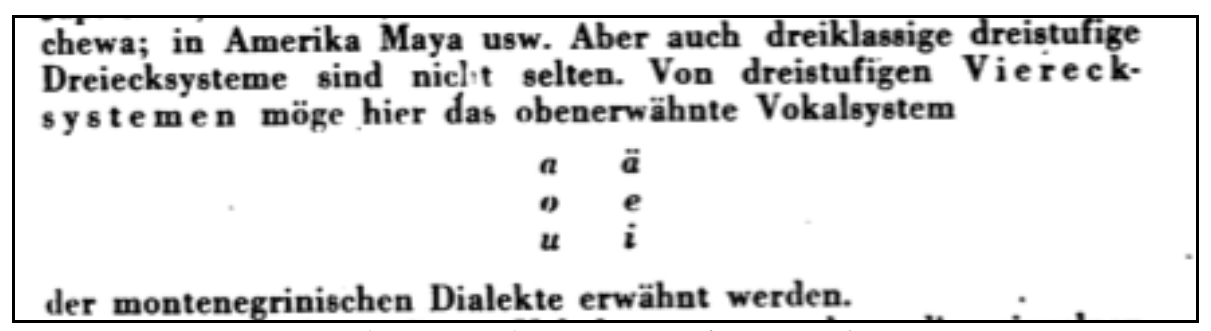

Texto de Trubetzkoy 1 (1939: 99)

La afirmación de que "se podría citar aquí de nuevo el sistema vocálico de los dialectos montenegrinos" no deja lugar a dudas de que, con esas escasas diez páginas de diferencia, se están empleando dos notaciones diferentes para hacer referencia al mismo objeto fonológico: $<æ>$ vs. $<a ̈>$. Dos notaciones que se unen a las ya mencionadas de Alarcos y Rešetar formando un cuarteto de sospechosos involucrados en el misterio de definir / describir un objeto que a priori se considera fonológico y que está presente en los sistemas de algunos dialectos montenegrinos. En el siguiente apartado de este trabajo, se propondrá una manera de analizar de manera unitaria los datos encontrados.

\section{Una unidad teórica para unificar el análisis de los datos, el glifo}

En esta investigación, hasta el momento, se está usando un corpus de obras científicas formado por: el Grundzüge der Phonolologie de Trubetzkoy en su versión original alemana (1939) y su traducción al francés efectuada por Cantineau (Trubetzkoy 1967) y al serbio por Berić y Berić (Trubeckoj 2016), el texto en español de la primera y la cuarta edición de la Fonología Española de Alarcos (1950, 1965), el texto original en alemán de Der stokavische Dialekt de Rešetar (1909) y la traducción al serbio (1994) de la obra de Ivić Die serbokroatichen Dialekte (1958). Estos siete textos forman el universo particular la dimensión gráfica de la notación científica, $\langle\mathrm{NC}\rangle$, del problema que se está analizando, la identificación de un determinado objeto fonológico (una vocal) en una serie de dialectos del serbo-croata (según la definición de los propios autores) hablados

${ }^{5}$ La traducción al francés de este texto de Cantineau dice: “Parmi les systèmes quadrangulaires à trois degrés on pourrait citer ici de nouveau le système vocalique [mismo esquema de vocales que en el original alemán] des dialectes monténégrins" (Trubetzkoy 1967). 
en Montenegro. Si se reunieran todos los artículos, monografías y libros que abordasen dicho problema dentro de un campo particular como el de dialectología del serbo-croata o de uno más general como sería el de la evolución o descripción fonológica de las lenguas eslavas se obtendría un universo completo de elementos de la $\langle\mathrm{NC}\rangle$ de este caso de estudio.

La TABLA 1 resume estos elementos de la $\langle N C\rangle$ investigada en este trabajo siguiendo un criterio cronológico (por fecha de publicación de la obra). El Grundzüge de Trubetzkoy presenta dos elementos (dos columnas) ya que, como se ha explicado anteriormente, ambos aparecen citados en la obra. Se ha respetado el uso de la cursiva como convención gráfica usada por todos los autores o editores.

\begin{tabular}{|c|c|c|c|c|}
\hline Resetar & Trubetzkoy & Trubetzkoy & Alarcos & Ivić \\
\hline $1907: 92$ & $1939: 89$ & $1939: 99$ & $1950: 40$ & $1994: 195$ \\
\hline$a^{e}$ & $æ$ & $\ddot{a}$ & $a e$ & $\ddot{a}$ \\
\hline
\end{tabular}

TABLA 1: Elementos de la $<\mathrm{NC}>$ de este trabajo

La primera propuesta de este trabajo es denominar a este cuarteto de elementos diferentes, $<\mathrm{a}^{\mathrm{e}}$, ä, $æ$, ae $>$, con el mismo término teórico glifo y usando la convención de notar los glifos entre corchetes angulares. De aceptarse dicha propuesta, la definición de glifo sería, en el contexto de una teoría general de la representación gráfica, la de unidad básica de la $\langle N C\rangle$. Frente a esta propuesta, se podría objetar que no sería necesario inventar un nuevo término, glifo, para dar cuenta de algo que se puede definir con el término tradicional de letra. Sin embargo, éste último no parece muy adecuado para describir el cuarteto de elementos que aparecen en la TABLA 1 ya que, en cada caso, habría que completarlo por medio de construcciones como, por ejemplo: "elemento formado por dos letras (ae); elemento formado por una letra y otra sobreescrita por encima de la línea $\left(a^{e}\right)$ o elemento formado por una letra y un diacrítico (ä)".

Además, no se puede olvidar, que el término letra se refiere a los elementos que forman parte de los sistemas gráficos de escritura basados en alfabetos, por lo que, técnicamente hablando, algunos de los elementos del cuarteto serían letras y otros no: ä sería una letra en las versiones del alfabeto latino que se usan, por ejemplo, para notar el alemán y æ sería lo mismo para el caso del islandés o el noruego; mientras que ae no sería una letra, sino una secuencia de dos letras, al igual que ocurre con $a^{e}$. Y esta última, aunque también sería una secuencia de dos letras (con una especial disposición espacial; en la línea y sobre la línea), no se emplea en la representación gráfica de ninguna de las lenguas que utilizan modificaciones del alfabeto latino, es un elemento de un metalenguaje descriptivo.

Por las razones expuestas, parece más adecuado utilizar un único término teórico, el glifo. La inspiración para este término viene de la obra de Heselwood (2013: 266) que define glifo (en inglés glyph) como "the graphic form of a character or symbol". Aunque para entender esta definición hay que tener en cuenta todo el sistema que se expondrá en 
el siguiente apartado. De igual manera, optar por este término general no significa renunciar a preguntas sobre los motivos por los que cada autor ha elegido un glifo u otro - sobre las realidades que se pretenden notar con cada uno de ellos, sino todo lo contrario: se pretende proporcionar una herramienta que haga más visible el análisis, separando claramente las diferentes capas de lenguajes y meta-lenguajes presentes en la formulación por escritos de las diferentes teorías fonéticas o fonológicas.

\section{El modelo propuesto y el desarrollo de un ejemplo, el caso de $a$}

El modelo que se propone pretende relacionar dos dimensiones a través de dos operaciones.

1) La primera dimensión es la dimensión gráfica de los textos y está formada por cualquier texto escrito en cualquiera de los sistemas de escrituras usados a través de la historia de la escritura - logosilabográfico, silabográfico, abjad, abugida, alfabético o de rasgos -. Esta primera dimensión se abrevia por medio del símbolo $\langle\mathrm{T}\rangle$ y la unidad mínima de la misma se denomina grafema. Se considerarían grafemas tanto los logogramas (usados en la escritura china), como los silabogramas (de la escritura japonesas) o las letras de cualquier alfabeto. Un ejemplo de una cadena de cinco (o seis) ${ }^{6}$ glifos en alfabeto latino es el nombre del autor de este trabajo, <César>. Dicha cadena presentaría cuatro glifos si se

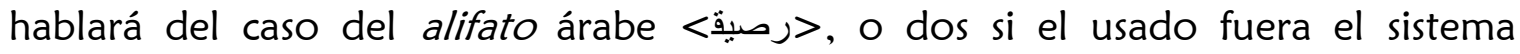
logosilabográfico chino, <凱撒>. La convención propuesta para representar cualquier grafema es utilizar corchetes angulares, $<>$, y las fuentes originales del sistema gráfico que se cite.

2) La otra dimensión es la expuesta inicialmente en este trabajo, la dimensión gráfica de la notación científica, $\langle\mathrm{NC}\rangle$, la forman todos los textos que reflexionan (por escrito) sobre cualquier elemento de la dimensión $\langle\mathrm{T}\rangle$. Como se ha explicado antes, la unidad de esta dimensión es el glifo. En este caso se usa la misma convención de emplear los corchetes angulares.

La relación entre las dos dimensiones descritas se hace por medio de dos operaciones:

3) La primera de ellas es la transliteración, abreviada TRaL. Una definición muy adecuada de la misma, aunque lleve el adjetivo "bibliográfico" debido al manual en el que aparece, es la siguiente de Wellisch (1978):

Bibliographic transliteration is the operation of converting the characters of a source script into the character of a target script. In principle, this is a one-to-one

${ }^{6}$ La diferencia entre contabilizar cinco o seis grafemas en la dimensión $<T>$ del español reside en si el glifo vocálico con tilde <é> se cuenta como uno o dos grafemas. 
transformation, in which one character of the source script is converted into one (and only one) specific character of the target script. (Wellisch 1978: 96)

La unidad mínima de la operación de TRaL es el carácter y es importante señalar que un carácter es la relación entre dos elementos cada uno de ellos perteneciente a un sistema gráfico diferente (el sistema gráfico fuente y el sistema gráfico meta). Es como un par ordenado en el que el primer elemento pertenece al primer sistema gráfico y el segundo al otro. En esquema: < elemento 1 : elemento $2>$. Usando el mismo ejemplo de antes, el del nombre del autor de este trabajo, la transliteración de la variante del alfabeto latino usada en el español a la variante del alfabeto cirílico usada en serbio sería las siguientes cadenas de caracteres relacionadas: <César : Цезар>.

4) La segunda es la transcripción, abreviada $\mathrm{TRaC}$. Esta última se divide, a su vez, en dos: transcripción fonética [TRaC] o fonológica /TRaC/. Mounin (1974: 327) define esta operación para los dos casos citados (incluyendo ejemplos).

Transcription - Représentation d'une séquence d'unités phoniques au moyen de caractères graphiques conventionnels. La transcription varie en fonction du but recherché. Elle peut ne noter que les unités phonétiques pertinentes du point de vue de la communication : transcription phonologique (entre barres obliques) bec de gaz /bekdgaz/ - ou bien les réalisations effectivement perçues (variantes contextuelles ou individuelles de ces unités comme l'ouverture du /e/, la sonorisation du $/ \mathrm{k} /$ ou la réalisation de la voyelle latente [ə] ): transcription phonétique (entre crochets carrés) [beķdə'gaz]. Dans se dernier cas, la transcription pourra être large (ang. broad) si elle n'indique que les caractéristiques générales des sons, ou a contraire étroite (angl. narrow) si elle est plus précise et détaillée. Mounin (1974: 327)

La unidad básica en la operación de la TRaC es el símbolo. La convención de utilizar corchetes cuadrangulares o barras sirve para distinguir símbolos que notan fonemas de aquellos que notan sonidos.

La TABLA 2 resume el modelo propuesto con sus diferentes dimensiones, operaciones, abreviaturas identificativas y unidades básicas.

\begin{tabular}{|l|c|c|}
\hline dimensión gráfica de los textos & $<\mathrm{T}\rangle$ & grafema \\
\hline operación transcripción fonética & {$[\mathrm{TRaC}]$} & símbolo \\
\hline operación transliteración & $\mathrm{TRaL}$ & carácter \\
\hline operación transcripción fonológica & /TRaCl & símbolo \\
\hline dimensión gráfica de la notación científica & $<\mathrm{NC}>$ & glifo \\
\hline
\end{tabular}

TABLA 2: Resumen del modelo

El objetivo del modelo es servir de herramienta para analizar las formas (cadenas de glifos) que aparecen en los textos que pueden configurar cualquier $<\mathrm{NC}>$. Por lo tanto, no se trata de un modelo fonológico, ni de uno fonético; ni es una guía para evaluar la validez de propuestas teóricas, o para efectuar transcripciones o 
transliteraciones. Sólo pretende servir para averiguar si se están comparando elementos correspondientes a un mismo nivel o a distintos niveles (lo que produciría problemas de interpretación). A continuación, en la TABLA 3, se ejemplifica el modelo propuesto con el caso de uno de los miembros del cuarteto citado, $\alpha$.

\begin{tabular}{|c|c|c|}
\hline & & EJEMPLOS Y COMENTARIOS \\
\hline$<\mathrm{T}>$ & $\begin{array}{c}\text { grafem } \\
\text { a }\end{array}$ & < bær > "baya" en la ortografía danesa, noruega o islandesa \\
\hline [TRaC] & símbolo & [æ] vocal casi abierta anterior no redondeada en el alfabeto de la AFI \\
\hline TRaL & carácter & < нæуæг : næuæg > transliteración cirílico - latino de "noche" en osetio. \\
\hline /TRaC/ & símbolo & $\begin{array}{l}\text { /æ/ near-open front unrounded vowel en el sistema fonológico inglés: "cat" } \\
\text { /kæt/. }\end{array}$ \\
\hline$<\mathrm{NC}$ & glifo & $<æ>$ glifo en el Grundzüge de Trubetzkoy. \\
\hline
\end{tabular}

TABLA 3: Ejemplificación del modelo para el caso de $a$

Desarrollando el ejemplo propuesto: un lector se puede encontrar el grafema $<æ>$, por ejemplo, en un periódico noruego y dependerá de su capacidad para leer ese idioma que pueda interpretarlo o no. También puede encontrar el glifo $\langle æ\rangle$ en un manual sobre la evolución de las lenguas germánicas y preguntarse (si el autor no lo ha explicitado previamente) si allí se está intentado representar el sonido [æ] o el fonema /æ/. Igualmente, si el mismo lector se encuentra una transliteración al alfabeto latino de un texto osetio, lengua que como sistema gráfico utiliza una variedad del alfabeto cirílico, verá que en el resultado de dicha operación hay caracteres que no varían ya que son comunes a ambos sistemas gráfico, como ocurre con el par $\langle æ: æ>$. La duda que le podría surgir al hipotético lector es saber a qué corresponde el glifo $\langle æ>$ en una obra de tipo teórico como puede ser la de Trubetzkoy y la relación que se puede establecer entre ese glifo y otros que puedan aparecer en otras obras, que es lo que ocurre precisamente en nuestro caso de estudio.

\section{El caso estudiado: el glifo <ae > en la obra de Alarcos}

Siguiendo lo expuesto hasta aquí, la conclusión sería que el glifo <ae> se está usando en la Fonología de Alarcos para notar un único fonema, que también se podría notar mediante el uso de otros como símbolos /æ/ o /ä/. Antes de entrar en la posible interpretación de esos símbolos u otros, hay que señalar que la identificación como fonema del objeto fónico representado por el glifo <ae> no es algo que se desprenda de la notación del párrafo citado, sino del contexto de la obra y del uso de la notación habitual (corchetes cuadrangulares y barras) que el propio autor también usa en las 
páginas iniciales de su obra como muestra este párrafo de la página 26 de la primera edición (1950):

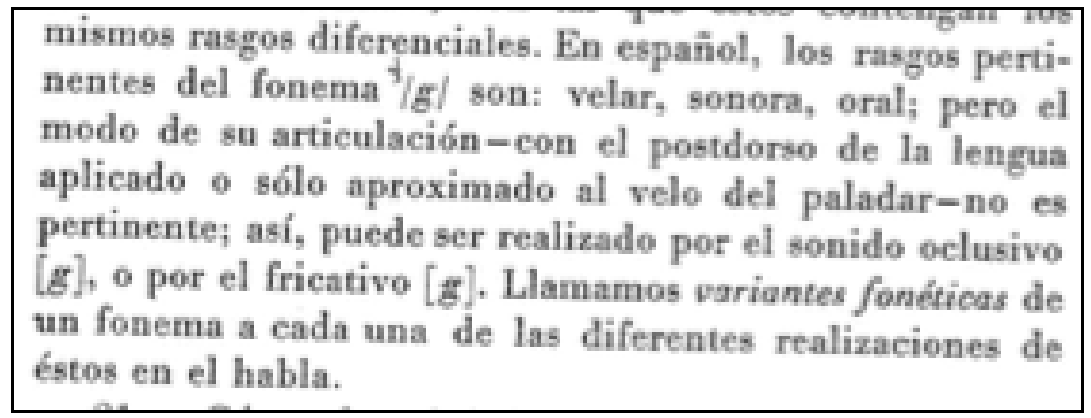

Texto de Alarcos 3: 1르 edición (1950: 27)

Aquí la oposición entre un fonema /g/ y un sonido [g] se marca por medio del uso, habitual ya en algunos textos de la época, de la convención citada (barras vs. corchetes angulares). Un uso muy relevante al tratarse de un texto pionero del campo de la fonología sobre el español (Matluck 1995).

Una descripción de dichos símbolos mencionados arriba sería la siguiente:

\begin{tabular}{|l|l|l|l|l|}
\hline$æ$ & vocal & casi abierta & anterior & no redondeada \\
\hline$a ̈$ & vocal & abierta & central & no redondeada \\
\hline
\end{tabular}

Antes de entrar en la naturaleza de lo que puede encontrarse bajo dichos símbolos, es necesario explicar que son diferentes dentro del propio alfabeto de la Asociación Fonética Internacional. El primero de ellos, a, es una de las 27 vocales que aparecen definidas en dicho alfabeto; mientras que el segundo es la combinación de un símbolo (a) más un diacrítico, los dos puntos, con el fin de enfatizar precisamente la "centralidad" de dicho fonema. Ahora bien, ipor cuál de los dos fonemas debemos decantarnos a la hora de interpretar el glifo del texto de Alarcos? Esta pregunta no tiene una respuesta fácil ya que, por ejemplo, ambos símbolos aparecen como glifos en la bibliografía citada ( $<\mathfrak{x}>$ en Trubetzkoy y $<a ̈>$ tanto en Trubetzkoy como en Ivić). Además, el hecho de que un mismo autor, Trubetzkoy, use ambos puede indicar su práctica equivalencia.

Aunque volviendo al glifo $<æ>$ en la obra de Trubetzkoy (1939: 103), también hay que observar que es usado para describir sistemas de otras lenguas, como por ejemplo del noruego. Un ejemplo que también recoge Alarcos (1950: 43; 1965: 63) con el mismo "problema" del uso del glifo $\langle a e\rangle$ y que resulta especialmente relevante debido a los diferentes niveles del modelo estudiados en el caso del noruego. Por ejemplo, en la $\langle\mathrm{T}>$ de esta lengua (como aparecía en la TABLA 3 citada arriba) aparece el grafema <ae> que, a nivel fonológico, podría tener interpretaciones diferentes 
susceptibles de ser notadas con los símbolos /æ/ o / $/$ /. Estudiar en profundidad la problemática del caso del noruego (a nivel fonológico o fonético) excede de los límites

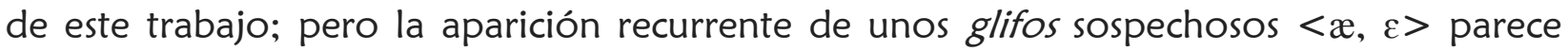
apuntar a la confusión de niveles en muchas descripciones. Esta pareja de sospechosos, por ejemplo, es la culpable de muchas explicaciones del colapso de cantidad en latín clásico y la diptongación de las vocales abiertas en las lenguas románicas. En ese último caso, su presencia se completa con la de otros glifos dando lugar a otro cuarteto muy repetido: $<æ$, ai, e, $\varepsilon>$.

Un último aspecto que hay que explicar del ejemplo estudiado con referencia al modelo propuesto es que, en este caso, lo que hay que analizar no es la relación entre una dimensión gráfica de los textos, $\langle\mathrm{T}\rangle$, con una dimensión gráfica de la notación científica $<\mathrm{NC}>$ creada por unos autores determinados usando una serie de glifos, sino el intento de estos de reflejar una dimensión fónica: se está hablando de unos dialectos montenegrinos que es muy probable que nunca se hayan escrito o que, si lo han sido, haya sido siguiendo las normas ortográficas marcadas por los estándares. En este sentido, quizá lo más fiable para intentar averiguar a qué fonema se podrían asignar los glifos estudiados sería intentar localizar hablantes de los dialectos en cuestión, si esto es posible más de un siglo después a las observaciones iniciales de Rešetar; o releer la obra de aquél intentando "traducir" sus observaciones (como la explicación de que se trata de "una vocal intermedia entre a y e») a una lengua-meta descriptiva que permita su comparación con objetos fónicos más que aparezcan en otros contextos.

Precisamente esto último es el objeto final del modelo propuesto, servir de instrumento para contextualizar textos de la $\langle\mathrm{NC}\rangle$ y poder diferenciar entre verdaderos problemas fonológicos o fonéticos y falsos problemas producidos por el uso de notaciones confusas. En este sentido, el glifo que aparece en todas las ediciones de la Fonologŕa es un caso de notación confusa, pues utiliza dos elementos $<a+$ e $>$ para notar una única unidad, un único objeto (en este caso, fonológico). Algo que, quizá no pudiera parecer tan problemático en otros niveles-como el de la representación ortográfica-, pero que tiene serías implicaciones en un texto con un objetivo teórico ya que se trata de ejemplo de meta-lenguaje fonológico.

Desde luego, se trata de un ejemplo mínimo que no resta de ninguna manera importancia a una obra tan fundamental y para el que se podrían buscar explicaciones de naturaleza tipográfica como, por ejemplo, que la editorial no dispusiese en ese momento del tipo adecuado para representar $\langle æ>$ (un argumento muy discutible tratándose de una editorial con tanta tradición en el mundo de la filología y la lingüística como la editorial española Gredos). Pero, en todo caso, la existencia de este ejemplo sirve para insistir en la necesidad de entender la $\langle N C\rangle$ de los textos como un objeto de estudio, en el que se identifican tradiciones investigadoras y costumbres de edición, a la vez que se separan claramente los elementos y niveles que coexisten en esa aparente superficie bidimensional, el texto escrito con intención científica. 


\section{BIBLIOGRAFÍA}

Alarcos Llorach, Emilio. Fonología Española. 1ª Edición. Madrid: Gredos, 1950. Impreso.

—. Fonología Española. 4a Edición, 8a Reimpresión. Madrid: Gredos, 1991. Impreso.

Calero, María Luisa, et al. (eds.) Métodos y resultados actuales en Historiografía de la Linguística. Münster: Nodus Publikationen, 2014. Impreso.

Heselwood, Barry. Phonetic transcription in theory and practice. Edinburgh: Edinburgh University Press, 2013. Print.

Ivić, Pavle. Die serbokroatischen Dialekte: ihre Struktur und Entwicklung. Mouton, 1958. Druck. Impreso.

-. Srpskohrvatski dijalekti: njihova struktura i razvoj. S nemačkog prevela Pavica Mrazović. [Traducido del alemán por Pavica Mrazović.] Sremski Karlovci / Novi Sad: Izdavačka knjižarnica Zorana Stojanovića, 1994. Štampano.

Matluck, Joseph G. “Emilio Alarcos Llorach, Fonología Española, 2ª . Ed. corregida y aumentada. Gredos, Madrid, 1954; 232 pp. (Biblioteca románica hispánica)". Nueva Revista de Filología Hispánica (NRFH), 9.3 (1995): 277-280. Impreso.

Mounin, Georges. Dictionnaire de la Linguistique. Paris: Presses Universitaires de France, 1974. Imprimé.

Perea, Francisco José. "Las cuatro ediciones de la Fonología española (1950-1965) de Emilio Alarcos". María Luisa Calero et al. (eds.), Métodos y resultados actuales en Historiografía de la Linguiística, Münster: Nodus Publikationen, 2014: 562-573. Impreso.

Rešetar, Milan. Der štokavische Dialekt. Wien, 1907. Druck.

Trubetzkoy, Nikolai. Grundzüge der phonologie. Praga: TCLP, 1939. Druck.

-. Principes de phonologie. Traducción al francés de Jean Cantineau. Paris: Librairie Klincksieck, 1967. Imprimé.

-. Principios de fonología. $1^{\text {a }}$ edición. Traducción al español de Delia García Giordano con la colaboración de Luis J. Prieto. Madrid: Editorial Cincel, 1973. Impreso.

Trubeckoj, Nikolaj Sergejevič [Trubetzkoy, Nikolai]. Načela fonologije. Prevele s nemačkog Vesna Berić i Andrijana Berić; redaktor prevoda Aleksandra Colić. [Traducido del alemán por Vesna Berić y Andrijana Berić. La traducción redactada por Aleksandra Colić] Sremski Karlovci; Novi Sad: Izdavačka knjižarnica Zorana Stojanovića, 2016. Štampano.

Wellisch, H. Hans. The Conversion of Scripts. Its Nature, History, and Utilization. New York: John Wiley \& Sons, Inc., 1978. Print. 